PROCEEDINGS OF THE

AMERICAN MATHEMATICAL SOCIETY

Volume 138, Number 4, April 2010, Pages 1405-1413

S 0002-9939(09)10164-8

Article electronically published on October 30, 2009

\title{
THE ERGODICITY OF WEAK HILBERT SPACES
}

\author{
RAZVAN ANISCA
}

(Communicated by Nigel J. Kalton)

\begin{abstract}
This paper complements a recent result of Dilworth, Ferenczi, Kutzarova and Odell regarding the ergodicity of strongly asymptotic $\ell_{p}$ spaces. We state this result in a more general form, involving domination relations, and we show that every asymptotically Hilbertian space which is not isomorphic to $\ell_{2}$ is ergodic. In particular, every weak Hilbert space which is not isomorphic to $\ell_{2}$ must be ergodic. Throughout the paper we construct explicitly the maps which establish the fact that the relation $E_{0}$ is Borel reducible to isomorphism between subspaces of the Banach spaces involved.
\end{abstract}

\section{INTRODUCTION}

The positive solution to the homogeneous Banach space problem, which was obtained by combining the Gowers dichotomy theorem [10] with results of Komorowski and Tomczak-Jaegermann [13, states that if a Banach space $X$ has only one class of isomorphism of infinite-dimensional subspaces, then $X$ must be isomorphic to $\ell_{2}$. It is therefore natural to investigate the complexity of the relation of isomorphism between subspaces of a given separable Banach space which is not isomorphic to $\ell_{2}$. In this context, let $E_{0}$ be the relation of eventual agreement of infinite binary sequences, defined on $\{0,1\}^{\mathbb{N}}$ by

$$
\alpha E_{0} \beta \Longleftrightarrow \exists m \forall n \geq m, \quad \alpha(n)=\beta(n) .
$$

As defined by Ferenczi and Rosendal [7, a separable Banach space $X$ is said to be ergodic if the relation $E_{0}$ is Borel reducible to isomorphism between subspaces of $X$, that is, if there exists a Borel map $f$ mapping elements of $\{0,1\}^{\mathbb{N}}$ to subspaces of $X$ such that

$$
\alpha E_{0} \beta \Longleftrightarrow f(\alpha) \simeq f(\beta) .
$$

It is clear that an ergodic Banach space $X$ must contain $2^{\omega}$ mutually non-isomorphic subspaces. For the general definition of the classification of equivalence relations by their complexity we refer the reader to $[5]$ and $[9]$.

Various results are known regarding ergodic spaces. It was observed in 17 that hereditarily indecomposable Banach spaces are ergodic, while Ferenczi and Rosendal proved in a series of papers [6], 7] that a non-ergodic Banach space $X$ with an unconditional basis must be isomorphic to its hyperplanes, to its square, and more generally to any direct sum $X \oplus Y$, where $Y$ is generated by a subsequence of the basis of $X$. Among the classical Banach spaces, $c_{0}$ and $\ell_{p}$, with $1 \leq p<2$,

Received by the editors May 29, 2009, and, in revised form, August 5, 2009.

2010 Mathematics Subject Classification. Primary 46B20; Secondary 46B15.

The author was supported in part by NSERC Grant 312594-05.

(C)2009 American Mathematical Society Reverts to public domain 28 years from publication 
are known to be ergodic (4), and the problem is still open for the other range of indices $p>2$. Also, as a consequence of their study of minimality for strongly asymptotic $\ell_{p}$ spaces [3], it was proved by Dilworth, Ferenczi, Kutzarova and Odell that for a Banach space $X$ with a strongly asymptotic $\ell_{p}$ basis either: $X$ is ergodic, or the basis of $X$ is equivalent to the unit vector basis of $\ell_{p}$. It was conjectured in [7] that every separable Banach space not isomorphic to $\ell_{2}$ must be ergodic.

In this paper we provide some additional support to this conjecture by proving that inside the regular class of weak Hilbert spaces every Banach space which is not isomorphic to $\ell_{2}$ is ergodic. We actually show that the same is true for the class of asymptotically Hilbertian spaces (Theorem 2.3). Another feature of the paper is that we construct explicitly the maps which establish the fact that the relation $E_{0}$ is Borel reducible to isomorphism between subspaces of the Banach spaces involved. In contrast, the work in [6], 7], 3] relies on set-theoretical arguments which provide the existence of such maps. With this in mind, in the first part of the paper we revisit the result of Dilworth, Ferenczi, Kutzarova and Odell and we prove (constructively) a more general version which involves domination relations and which is of independent interest (Theorem 2.1).

We finish the section with some notation essential for our argument. We refer the reader to 14 for all terminology not explained here. In particular, the fundamental concept of a basis and a Schauder decomposition can be found in 14, 1.a.1 and 1.g.1, respectively.

For $C \geq 1$, a basis $\left\{e_{i}\right\}_{i}$ in a Banach space $X$ is called $C$-unconditional if for every $x=\sum_{i} a_{i} e_{i} \in X$ one has $\left\|\sum_{i} \epsilon_{i} a_{i} e_{i}\right\| \leq C\|x\|$, for all signs $\epsilon_{i}= \pm 1$.

Let $\left\{e_{i}\right\}_{i}$ and $\left\{u_{i}\right\}_{i}$ be two infinite basic sequences. We say that $\left\{e_{i}\right\}_{i}$ and $\left\{u_{i}\right\}_{i}$ are $C$-equivalent if there exist constants $A, B>0$ with $A B \leq C$ and such that

$$
(1 / A)\left\|\sum_{i} a_{i} e_{i}\right\| \leq\left\|\sum_{i} a_{i} u_{i}\right\| \leq B\left\|\sum_{i} a_{i} e_{i}\right\|
$$

for all finite sequences of scalars $\left\{a_{i}\right\}_{i}$.

We say that $\left\{e_{i}\right\}_{i}$ dominates (respectively is dominated by) $\left\{u_{i}\right\}_{i}$ if there exists $B<\infty$ (respectively, $A>0$ ) such that the right (respectively left) inequality in (1.1) is satisfied. By $I: \overline{\operatorname{span}}\left\{e_{i}\right\}_{i} \longrightarrow \overline{\operatorname{span}}\left\{u_{i}\right\}_{i}$ we denote the formal identity operator, that is, $I(x)=\sum_{i} a_{i} u_{i}$ for $x=\sum_{i} a_{i} e_{i}$.

Let $1 \leq p \leq \infty$. A Banach space $X$ with a basis $\left\{e_{i}\right\}_{i}$ is strongly asymptotic $\ell_{p}$ if there exist $C<\infty$ and an increasing function $f: \mathbb{N} \longrightarrow \mathbb{N}$ such that, for all $n \in \mathbb{N}$, every normalized sequence $\left\{x_{i}\right\}_{i=1}^{n}$ of disjointly supported vectors from $\overline{\operatorname{span}}\left\{e_{i}\right\}_{i=f(n)}^{\infty}$ is $C$-equivalent to the unit vector basis of $\ell_{p}^{n}$.

If we only consider domination relations above, we can say that a Banach space $X$ with a basis $\left\{e_{i}\right\}_{i}$ admits asymptotically a lower p-estimate on disjoint vectors if there are $C<\infty$ and an increasing function $f: \mathbb{N} \longrightarrow \mathbb{N}$ such that, for all $n \in \mathbb{N}$ and normalized disjointly supported vectors $\left\{x_{i}\right\}_{i=1}^{n}$ from $\overline{\operatorname{span}}\left\{e_{i}\right\}_{i=f(n)}^{\infty},\left\{x_{i}\right\}_{i=1}^{n}$ dominates the unit vector basis of $\ell_{p}^{n}$ (with constant $C$ ). The situation when $\left\{e_{i}\right\}_{i}$ admits asymptotically an upper p-estimate on disjoint vectors can be defined in a similar manner.

Finally, given a Banach space $X$, a natural number $n$, and $1 \leq p \leq 2$ (respectively $2 \leq q<\infty$ ) we denote by $T_{p}(X, n)$ (respectively $\left.C_{q}(X, n)\right)$ the smallest constant $C$ 
such that

$$
\begin{gathered}
\int_{0}^{1}\left\|\sum_{i=1}^{n} r_{i}(t) x_{i}\right\| d t \leq C\left(\sum_{i=1}^{n}\left\|x_{i}\right\|^{p}\right)^{1 / p} \\
\left(\text { respectively } \quad C \int_{0}^{1}\left\|\sum_{i=1}^{n} r_{i}(t) x_{i}\right\| d t \geq\left(\sum_{i=1}^{n}\left\|x_{i}\right\|^{q}\right)^{1 / q}\right)
\end{gathered}
$$

for all $x_{1}, \ldots, x_{n} \in X$. Recall that $\left\{r_{i}\right\}_{i}$ are the Rademacher functions. When $T_{p}(X):=\sup _{n} T_{p}(X, n)<\infty$ we say that $X$ has type $p$, while if $C_{q}(X):=$ $\sup _{n} C_{q}(X, n)<\infty$ we say that $X$ has cotype $q$.

We can consider these properties in the asymptotic context as well. For $1 \leq p \leq 2$ (respectively $2 \leq q<\infty$ ) we say that a Banach space $X$ with a basis $\left\{e_{i}\right\}_{i}$ is asymptotically of type $p$ (respectively asymptotically of cotype $q$ ) if there exist $C<$ $\infty$ and an increasing function $f: \mathbb{N} \longrightarrow \mathbb{N}$ such that $T_{p}\left(\overline{\operatorname{span}}\left\{e_{i}\right\}_{i=f(n)}^{\infty}, n\right) \leq C$ (respectively $C_{q}\left(\overline{\operatorname{span}}\left\{e_{i}\right\}_{i=f(n)}^{\infty}, n\right) \leq C$ ) for all $n \in \mathbb{N}$. As an example, consider $q \geq 2$ and a sequence $s_{n} \searrow q$, along with a sequence of positive integers $\left\{k_{n}\right\}_{n}$ converging to $\infty$ sufficiently rapidly, say $k_{n}^{1 / q-1 / s_{n}} \nearrow \infty$. It is not hard to check that the Banach space

$$
X=\left(\sum_{n} \oplus \ell_{s_{n}}^{k_{n}}\right)_{\ell_{q}},
$$

is asymptotically of cotype $q$ while it does not admit cotype $q$.

\section{The MAIN CONSTRUCTIONS}

We begin by proving a more general version of the result from [3].

Theorem 2.1. Let $X$ be a Banach space with an unconditional basis $\left\{e_{i}\right\}_{i}$.

(i) Assume there is $1 \leq p \leq 2$ such that $X$ is asymptotically of type $p$, while the basis $\left\{e_{i}\right\}_{i}$ is not dominated by the unit vector basis of $\ell_{p}$. Then $X$ is ergodic.

(ii) Assume there is $2<q \leq \infty$ such that $X$ admits asymptotically an upper $q$-estimate on disjoint vectors, while the basis $\left\{e_{i}\right\}_{i}$ is not dominated by the unit vector basis of $\ell_{q}$ (or $c_{0}$ if $q=\infty$ ). Then $X$ is ergodic.

(iii) Assume there is $2 \leq q<\infty$ such that $X$ is asymptotically of cotype $q$, while the basis $\left\{e_{i}\right\}_{i}$ does not dominate the unit vector basis of $\ell_{q}$. Then $X$ is ergodic.

(iv) Assume there is $1 \leq p<2$ such that $X$ admits asymptotically a lower $p$ estimate on disjoint vectors, while the basis $\left\{e_{i}\right\}_{i}$ does not dominate the unit vector basis of $\ell_{p}$. Then $X$ is ergodic.

Proof. We outline only the proofs of $(i)$ and (ii) since the arguments in (iii) and (iv) are similar. We may assume that the basis $\left\{e_{i}\right\}_{i}$ is 1 -unconditional. We will construct inductively finite dimensional subspaces $\left\{F_{n}\right\}_{n}$ spanned by disjoint subsets of $\left\{e_{i}\right\}_{i}$ such that, whenever $\alpha, \beta \in\{0,1\}^{\mathbb{N}}$ are not $E_{0}$-equivalent,

$$
X(\alpha)=\sum_{j: \alpha(j)=1} \oplus F_{j} \text { and } X(\beta)=\sum_{j: \beta(j)=1} \oplus F_{j}
$$

are not isomorphic. If one defines $g(\alpha)=X(\alpha)$, for all $\alpha \in\{0,1\}^{\mathbb{N}}$, this map establishes that the relation $E_{0}$ is Borel reducible to isomorphism between subspaces of $X$. 
Denote by $\left\{u_{i}\right\}_{i}$ the unit vector basis of $\ell_{p}$ (in $\left.(i)\right)$, respectively $\ell_{q}$ (in $(i i)$ ). In addition, for a finite dimensional subspace $F=\operatorname{span}\left\{e_{i}\right\}_{i \in I}$ we will use the notation $|F|:=|I|=\operatorname{dim} F$.

(i) Assume that $X$ is asymptotically of type $p$ with constant $C$ and function $f$. Define $F_{1}=\operatorname{span}\left\{e_{i}\right\}_{s_{1} \leq i \leq t_{1}}$, where $s_{1}=1$ and $t_{1}$ satisfies $\| I: \operatorname{span}\left\{u_{i}\right\}_{i \leq t_{1}}$ $\longrightarrow \operatorname{span}\left\{e_{i}\right\}_{i \leq t_{1}} \| \geq 1$. Let $n \geq 2$ and assume that $F_{1}, F_{2}, \ldots, F_{n-1}$ have been constructed. If $F_{n-1}=\operatorname{span}\left\{e_{i}\right\}_{s_{n-1} \leq i \leq t_{n-1}}$, pick $s_{n}>\max \left\{t_{n-1}, f\left(\left|F_{n-1}\right|\right)\right\}$ and $t_{n}$ such that

$$
\left\|I: \operatorname{span}\left\{u_{i}\right\}_{s_{n} \leq i \leq t_{n}} \longrightarrow \operatorname{span}\left\{e_{i}\right\}_{s_{n} \leq i \leq t_{n}}\right\| \geq n\left(\left|F_{1}\right|+\ldots+\left|F_{n-1}\right|\right)^{1 / 2}
$$

and then let $F_{n}=\operatorname{span}\left\{e_{i}\right\}_{s_{n} \leq i \leq t_{n}}$. This is possible since the basis $\left\{e_{i}\right\}_{i}$ (and consequently $\left.\left\{e_{i}\right\}_{i \geq s_{n}}\right)$ is not dominated by the unit vector basis $\left\{u_{i}\right\}_{i}$ of $\ell_{p}$.

Assume that $X(\alpha)$ is $K$-isomorphic to $X(\beta)$ for some $\alpha, \beta \in\{0,1\}^{\mathbb{N}}$ which are not $E_{0}$-equivalent, and without loss of generality let $n$ be such that $\alpha(n)=1$ and $\beta(n)=0$. Then $F_{n}$ is $K$-isomorphic to a subspace of $X(\beta)=\left(\sum_{j<n: \beta(j)=1} \oplus F_{j}\right) \oplus$ $\left(\sum_{j>n: \beta(j)=1} \oplus F_{j}\right)$. By construction and the asymptotic type $p$ hypothesis, it is clear that we have $T_{p}\left(\sum_{j>n: \beta(j)=1} \oplus F_{j},\left|F_{n}\right|\right) \leq C$. On the other hand, we get $T_{p}\left(\sum_{j<n: \beta(j)=1} \oplus F_{j}\right) \leq c_{1}\left(\left|F_{1}\right|+\ldots+\left|F_{n-1}\right|\right)^{1 / 2}$ and therefore

$$
T_{p}\left(X(\beta),\left|F_{n}\right|\right) \leq c_{2} C\left(\left|F_{1}\right|+\ldots+\left|F_{n-1}\right|\right)^{1 / 2},
$$

where $c_{1}, c_{2}$ depend only on $p$.

Taking into account that the basis $\left\{e_{i}\right\}_{s_{n} \leq i \leq t_{n}}$ of $F_{n}$ is 1-unconditional we conclude that

$$
\begin{aligned}
\left\|\sum_{s_{n} \leq i \leq t_{n}} a_{i} e_{i}\right\| & \leq K T_{p}\left(X(\beta),\left|F_{n}\right|\right)\left(\sum_{s_{n} \leq i \leq t_{n}}\left|a_{i}\right|^{p}\right)^{1 / p} \\
& \leq c_{2} C K\left(\left|F_{1}\right|+\ldots+\left|F_{n-1}\right|\right)^{1 / 2}\left(\sum_{s_{n} \leq i \leq t_{n}}\left|a_{i}\right|^{p}\right)^{1 / p}
\end{aligned}
$$

for all choices of scalars $\left\{a_{i}\right\}_{s_{n} \leq i \leq t_{n}}$. For $n$ large enough this contradicts (2.1).

(ii) Assume that $X$ admits asymptotically an upper $q$-estimate on disjoint vectors with constant $C$ and function $f$. We slightly change the inductive construction of the subspaces $\left\{F_{n}\right\}_{n \geq 1}$ : we now choose $F_{n}=\operatorname{span}\left\{e_{i}\right\}_{s_{n} \leq i \leq t_{n}}$ with $s_{n}>$ $\max \left\{t_{n-1}, f\left(\left|F_{n-1}\right|\right)\right\}$ and

$$
\left\|I: \operatorname{span}\left\{u_{i}\right\}_{s_{n} \leq i \leq t_{n}} \longrightarrow \operatorname{span}\left\{e_{i}\right\}_{s_{n} \leq i \leq t_{n}}\right\| \geq n\left(\left|F_{1}\right|+\ldots+\left|F_{n-1}\right|\right)^{1-1 / q} .
$$

Assume that $X(\alpha) \simeq X(\beta)$ for certain $\alpha, \beta \in\{0,1\}^{\mathbb{N}}$ which are not $E_{0}$-equivalent. It is clear that the canonical basis of $X(\alpha)=\sum_{j: \alpha(j)=1} \oplus F_{j}$, as a subsequence of $\left\{e_{i}\right\}_{i}$, is 1-unconditional and the same is true for the canonical basis of $X(\beta)$. Since $2<q \leq \infty$ and $X$ admits asymptotically an upper $q$-estimate on disjoint vectors we deduce that each of these bases is lattice anti-Euclidean; that is, there is no constant $L$ with the property that $X(\alpha)$ (or $X(\beta)$ ) contains $L$-complemented, $L$-isomorphic copies of $\ell_{2}^{n}$ 's whose bases are disjointly supported on the basis of $X(\alpha)$. We refer the reader to [2] for the general definition in the lattice setting. By [2, Theorem 3.5], there exist $K>0$ and a positive integer $N$ such that the canonical basis of $\sum_{i: \alpha(i)=1, \beta(i)=0} \oplus F_{i}$ is $K$-equivalent to a sequence of disjointly supported vectors in $(X(\beta))^{N}$. The space $(X(\beta))^{N}$ should be viewed as 
a subspace of $X^{N}$, which is equipped with the norm $\left\|\left(x_{i}\right)_{i=1}^{N}\right\|=\max _{1 \leq i \leq N}\left\|x_{i}\right\|$ and has the canonical basis obtained from the basis $\left\{e_{i}\right\}_{i}$ of $X$ with the ordering $\left(e_{1}, 0, \ldots, 0\right),\left(0, e_{1}, 0, \ldots, 0\right), \ldots,\left(0, \ldots, 0, e_{1}\right),\left(e_{2}, 0, \ldots, 0\right)$, etc. This phenomenon is part of the more general technical result [2, Theorem 3.6], which contains additional information on the uniformity of the constants involved and which plays an essential role in [3].

Let $n$ be such that $\alpha(n)=1$ and $\beta(n)=0$. The basis $\left\{e_{i}\right\}_{s_{n} \leq i \leq t_{n}}$ of $F_{n}$ is $K$-equivalent to a sequence of disjointly supported vectors $\left\{w_{i}\right\}_{s_{n} \leq i \leq t_{n}}$ in

$$
(X(\beta))^{N}=\left(\sum_{j<n: \beta(j)=1} \oplus F_{j}\right)^{N} \oplus\left(\sum_{j>n: \beta(j)=1} \oplus F_{j}\right)^{N} .
$$

Denote by $J=\left\{i \mid w_{i} \in\left(\sum_{j>n: \beta(j)=1} \oplus F_{j}\right)^{N}\right\}$ and $J^{\prime}=\left\{s_{n} \leq i \leq t_{n}\right\} \backslash J$. On the one hand, taking into account that $\left|J^{\prime}\right| \leq N\left(\left|F_{1}\right|+\ldots+\left|F_{n-1}\right|\right)$ we can write

$$
\begin{aligned}
\left\|\sum_{i \in J^{\prime}} a_{i} e_{i}\right\| & \leq\left|J^{\prime}\right|^{1-1 / q}\left(\sum_{i \in J^{\prime}}\left|a_{i}\right|^{q}\right)^{1 / q} \\
& \leq N^{1-1 / q}\left(\left|F_{1}\right|+\ldots+\left|F_{n-1}\right|\right)^{1-1 / q}\left(\sum_{i \in J^{\prime}}\left|a_{i}\right|^{q}\right)^{1 / q}
\end{aligned}
$$

for all scalars $\left\{a_{i}\right\}_{i \in J^{\prime}}$. On the other hand, it is easy to see that $X^{N}$ admits asymptotically an upper $q$-estimate on disjoint vectors with constant $C^{\prime}$ depending on $N$ and $C$ and function $f^{\prime}: \mathbb{N} \longrightarrow \mathbb{N}, f^{\prime}(m)=N f(m)$ for all $m \in \mathbb{N}$. By construction we have $s_{n+1} \geq f\left(\left|F_{n}\right|\right)$ and thus we conclude that

$$
\begin{aligned}
\left\|\sum_{i=s_{n}}^{t_{n}} a_{i} e_{i}\right\| & \leq\left\|\sum_{i \in J} a_{i} e_{i}\right\|+\left\|\sum_{i \in J^{\prime}} a_{i} e_{i}\right\| \leq K\left\|\sum_{j \in J} a_{i} w_{i} /\right\| w_{i}\|\|+\left\|\sum_{i \in J^{\prime}} a_{i} e_{i}\right\| \\
& \leq K C^{\prime}\left(\sum_{i \in J}\left|a_{i}\right|^{q}\right)^{1 / q}+N^{1-1 / q}\left(\left|F_{1}\right|+\ldots+\left|F_{n-1}\right|\right)^{1-1 / q}\left(\sum_{i \in J^{\prime}}\left|a_{i}\right|^{q}\right)^{1 / q}
\end{aligned}
$$

for all choices of scalars $\left\{a_{i}\right\}_{s_{n} \leq i \leq t_{n}}$. For $n$ large enough this contradicts (2.2).

Theorem 2.1 now provides a constructive way of obtaining the result in [3].

Corollary 2.2. Let $X$ be a Banach space with a strongly asymptotic $\ell_{p}$ basis $\left\{e_{i}\right\}_{i}$. Assume that $\left\{e_{i}\right\}_{i}$ is not equivalent to the unit vector basis of $\ell_{p}$ (or $c_{0}$ if $p=\infty$ ). Then $X$ is ergodic.

Proof. The basis $\left\{e_{i}\right\}_{i}$ is unconditional and one of the following holds: $\left\{e_{i}\right\}_{i}$ is not dominated by the unit vector basis of $\ell_{p}$, or $\left\{e_{i}\right\}_{i}$ does not dominate the unit vector basis of $\ell_{p}$.

Assuming that the first alternative is true, Theorem 2.1 $(i i)$ provides the conclusion when $2<p$. In the case when $1 \leq p \leq 2$ the result is a consequence of Theorem 2.1 $(i)$. Indeed, suppose that $\left\{e_{i}\right\}_{i}$ is strongly asymptotic $\ell_{p}$ with constant $C$ and function $f$. Let $n \geq 1$ and $E \subseteq \overline{\operatorname{span}}\left\{e_{i}\right\}_{i \geq f\left(n^{2 n}\right)}$ be an $n$-dimensional subspace. According to the result of Johnson [11], $E$ 2-embeds into a subspace spanned by $n^{2 n}$ disjointly supported vectors in $\overline{\operatorname{span}}\left\{e_{i}\right\}_{i \geq f\left(n^{2 n}\right)}$, so $E$ is 2C-isomorphic to a 
subspace of $\ell_{p}^{n^{2 n}}$. Consequently $T_{p}(E) \leq 2 C c$, where $c$ is an absolute constant depending on $p$, and thus $X$ is asymptotically of type $p$.

We now turn our attention to another class of Banach spaces which we can show to be ergodic, namely the asymptotically Hilbertian spaces. Given integers $n \geq 0$, $m \geq 1$ and a constant $K$, we say that a Banach space $X$ satisfies $H(n, m, K)$ provided there is an $n$-codimensional subspace $Y$ of $X$ such that every subspace $E$ of $Y$ with $\operatorname{dim} E \leq m$ is $K$-isomorphic to $\ell_{2}^{\operatorname{dim} E}$. A Banach space $X$ is said to be asymptotically Hilbertian provided there is a constant $K$ so that for every $m$ there exists an $n$ such that $X$ satisfies $H(n, m, K)$. Compared to the other asymptotic structures considered in this paper, the property of being asymptotically Hilbertian does not assume that the Banach space $X$ admits a basis. In fact, there are examples of asymptotically Hilbertian spaces which fail the approximation property ([1]) .

Next we discuss another property which is closely related to $H(\cdot)$ and which will play an important part in the arguments. Namely, given integers $n \geq 0, m \geq 1$ and a constant $K$, we say that a Banach space $X$ satisfies $C(n, m, K)$ provided there is an $n$-codimensional subspace $Y$ of $X$ such that every subspace $E$ of $Y$ with $\operatorname{dim} E \leq m$ is the range of a projection $P$ from $X$ with $\|P\| \leq K$. When $X$ is a Banach space of type 2 and has the property $H(n, m, K)$, then, by Maurey's extension theorem, $X$ also satisfies $C\left(n, m, K T_{2}(X)\right)$. It was proved by Johnson [12, Proposition 1.8] that for general Banach spaces these two notions are in duality. In fact, if $X$ has $H(n, m, K)$, then $X^{*}$ has $H\left(5^{n}, \log _{5} m, 12 K\right)$ and $C\left(5^{n}, \log _{5} m, 12 K\right)$. Hence, since these notions are hereditary, this shows that the $H(\cdot)$ property implies the $C(\cdot)$ property even for spaces $X$ which are not of type 2: if $X$ has $H(n, m, K)$, then it also satisfies $C\left(5^{5^{n}}, \log _{5} \log _{5} m, 144 K\right)$.

Theorem 2.3. Let $X$ be an asymptotically Hilbertian space which is not isomorphic to $\ell_{2}$. Then $X$ is ergodic.

Proof. According to Johnson's result we can assume that there is a constant $K$ and, for every $m$, there are finite-codimensional subspaces $Z_{m}$ and $\tilde{Z}_{m}$ of $X$ such that every subspace $E$ of $Z_{m}$ (respectively of $\tilde{Z}_{m}$ ) with $\operatorname{dim} E \leq m$ is $K$-isomorphic to $\ell_{2}^{\operatorname{dim} E}$ (respectively is the range of a projection $P$ from $X$ with $\|P\| \leq K$ ).

We will construct inductively finite-dimensional subspaces $\left\{F_{n}\right\}_{n \geq 1}$ of $X$ with the following properties:

(i) $\left\{F_{n}\right\}_{n \geq 1}$ generates a Schauder decomposition for its span $\overline{\bigcup_{n}\left(F_{1}+\ldots+F_{n}\right)}$;

(ii) $d\left(F_{n}\right)>n\left(\left|F_{1}\right|+\ldots\left|F_{n-1}\right|\right)^{1 / 2}$, for all $n \geq 2$. Here we denote by $|F|:=$ $\operatorname{dim} F$ and by $d(F)$ the Banach-Mazur distance between $F$ and a Hilbert space of corresponding dimension;

(iii) any $\left|F_{n}\right|$-dimensional subspace of $F_{n+1} \oplus F_{n+2} \oplus \ldots$ is $K$-isomorphic to $\ell_{2}^{\left|F_{n}\right|}$, for all $n \geq 1$;

(iv) any $\left|F_{n}\right|$-dimensional subspace of $F_{n+1} \oplus F_{n+2} \oplus \ldots$ is the range of a projection $P$ from $X$ with $\|P\| \leq K$, for all $n \geq 1$.

In order to achieve this, the inductive procedure includes also the construction of finite-dimensional subspaces $\left\{Y_{n}\right\}_{n \geq 0}$ of $X^{*}$ such that, for all $n \geq 1$,

(v) $Y_{n-1} \subseteq Y_{n}$ and $F_{n} \subseteq Y_{n-1}^{\perp}$;

(vi) $Y_{n}$ is 2-norming over $\sum_{i=1}^{n} F_{i}$, that is, for all $x \in \sum_{i=1}^{n} F_{i}$,

$$
\|x\| \leq 2 \sup \left\{\left|x^{*}(x)\right| \mid x^{*} \in Y_{n},\left\|x^{*}\right\| \leq 1\right\}
$$

(vii) $Y_{n}^{\perp} \subseteq Z_{\left|F_{n}\right|}$ and $Y_{n}^{\perp} \subseteq \tilde{Z}_{\left|F_{n}\right|}$. 
Set $Y_{0}=\{0\}$ and take $F_{1}$ to be a finite-dimensional subspace of $X$ with $d\left(F_{1}\right)>$ 1. The choice of $Y_{1}$ is a consequence of the following well known and standard fact: if $E$ is a subspace of $X$ with $\operatorname{dim} E=l$, then there exists a $5^{l}$-dimensional subspace $Y \subseteq X^{*}$ which is 2-norming over $E$. Consider a finite-dimensional subspace $Y_{1}^{\prime} \subseteq$ $X^{*}$ which is 2-norming over $F_{1}$ and set $Y_{1}=Y_{1}^{\prime}+Z_{\left|F_{1}\right|}^{\perp}+\tilde{Z}_{\left|F_{1}\right|}^{\perp}$.

Assume now that $n \geq 1$ and $F_{1}, \ldots, F_{n}, Y_{0}, Y_{1}, \ldots, Y_{n}$ have been constructed to satisfy (ii), (v), (vi) and (vii). Since $Y_{n}^{\perp}$ is finite-codimensional and $X$ is not isomorphic to $\ell_{2}$ we can find a finite-dimensional subspace $F_{n+1} \subseteq Y_{n}^{\perp}$ with $d\left(F_{n+1}\right)>$ $(n+1)\left(\left|F_{1}\right|+\ldots+\left|F_{n}\right|\right)^{1 / 2}$. Take a finite-dimensional subspace $Y_{n+1}^{\prime} \subseteq X^{*}$ which is 2-norming over $\sum_{i=1}^{n+1} F_{i}$ and then let $Y_{n+1}=Y_{n}+Y_{n+1}^{\prime}+Z_{\left|F_{n+1}\right|}^{\perp}+\tilde{Z}_{\left|F_{n+1}\right|}^{\perp}$. Now that the inductive construction is completed it is easy to see that (v) and (vi) imply (i), while (iii) and (iv) are consequences of (v) and (vii).

In order to prove that $X$ is ergodic it remains to show, as in Theorem 2.1, that

$$
X(\alpha)=\sum_{j: \alpha(j)=1} \oplus F_{j} \quad \text { and } \quad X(\beta)=\sum_{j: \beta(j)=1} \oplus F_{j}
$$

are not isomorphic whenever $\alpha, \beta \in\{0,1\}^{\mathbb{N}}$ are not $E_{0}$-equivalent.

Assume there exists an isomorphism $T: X(\alpha) \rightarrow X(\beta)$, for some $\alpha, \beta \in\{0,1\}^{\mathbb{N}}$ which are not $E_{0}$-equivalent, and let $\tilde{K}>0$ satisfy $\|T\|\left\|T^{-1}\right\| \leq \tilde{K}$. Let $n$ be such that $\alpha(n)=1$ and $\beta(n)=0$. Then $F_{n}$ is $\tilde{K}$-isomorphic to a subspace of $X(\beta)=\left(\sum_{j<n: \beta(j)=1} \oplus F_{j}\right) \oplus\left(\sum_{j>n: \beta(j)=1} \oplus F_{j}\right)$. Put

$$
G=\sum_{j>n: \beta(j)=1} \oplus F_{j}, \quad E=T^{-1}\left(T\left(F_{n}\right) \cap G\right) .
$$

By (iv) we know that $T\left(F_{n}\right) \cap G$ is $K$-complemented in $X$, and thus in $X(\beta)$. Hence $E$ is the range of a projection $P$ on $F_{n}$ with $\|P\| \leq K \tilde{K}$. Denoting by $E^{\prime}=P^{-1}(0)$ we obtain $F_{n}=E \oplus E^{\prime}$ and

$$
\operatorname{dim} E^{\prime} \leq \sum_{j<n: \beta(j)=1}\left|F_{j}\right| \leq\left|F_{1}\right|+\ldots+\left|F_{n-1}\right| .
$$

Since by (iii) we have $d(E) \leq \tilde{K} d\left(T\left(F_{n}\right) \cap G\right) \leq \tilde{K} K$, as a result we obtain

$$
d\left(F_{n}\right) \leq 2 K \tilde{K}(1+K \tilde{K}) d\left(E^{\prime}\right) \leq 2 K \tilde{K}(1+K \tilde{K})\left(\left|F_{1}\right|+\ldots+\left|F_{n-1}\right|\right)^{1 / 2} .
$$

For $n$ large enough this contradicts (ii).

As a consequence of Theorem 2.3 we obtain that every weak Hilbert space which is not isomorphic to $\ell_{2}$ is ergodic. A Banach space $X$ is a weak Hilbert space provided that there are positive constants $\delta$ and $K$ such that for every $n$, every $n$-dimensional subspace of $X$ contains a further subspace $E$ of dimension at least $\delta n$ with the property that $E$ is $K$-isomorphic to a Hilbert space and $E$ is $K$ complemented in $X$. The original definition of a weak Hilbert space is different and the characterization mentioned above is chosen out of many equivalent properties proved by Pisier [15]. Of importance for us is the fact that a weak Hilbert space is asymptotically Hilbertian (see [15], Section 4).

Corollary 2.4. Let $X$ be a weak Hilbert space which is not isomorphic to $\ell_{2}$. Then $X$ is ergodic. 
The canonical example of a weak Hilbert space which is not a Hilbert space is $T^{(2)}$, the 2-convexification of Tsireslon's space (most precisely, of the space $T$ introduced by Figiel and Johnson in [8]). In terms of the complexity of the relation of isomorphism between subspaces, for $T^{(2)}$ a stronger result than Corollary 2.4 was already proved by Rosendal [18.

The proof of Theorem 2.3 also shows that the relation $E_{0}$ is Borel reducible to uniform homeomorphism between subspaces of $X$ when $X$ is asymptotically Hilbertian (and the same is true for the space $X$ in Theorem $2.1(i)$ and (iii)). Recall that Banach spaces $Y$ and $Z$ are uniformly homeomorphic if there exists a uniformly continuous bijection $\phi: Y \longrightarrow Z$ such that $\phi^{-1}$ is also uniformly continuous. The fact that $E_{0}$ is Borel reducible to uniform homeomorphism between infinite-dimensional subspaces of $X$ is a consequence of a fundamental theorem of Ribe [16] which asserts that the local structure of finite-dimensional subspaces is invariant under uniform homeomorphisms: if $Y$ and $Z$ are uniformly homeomorphic Banach spaces, then there exists a constant $C>0$ such that for every finitedimensional subspace $E$ of $Y$ there exists a finite-dimensional subspace $F$ of $Z$ such that $d(E, F) \leq C$, and vice versa. In our situation, in the proof of Theorem 2.3 we concluded that the subspaces $X(\alpha)$ and $X(\beta)$ are not isomorphic, when $\alpha, \beta \in$ $\{0,1\}^{\mathbb{N}}$ are not $E_{0}$-equivalent, based on the fact that given any $\tilde{K}>0$ there exists an $n$ with the property that $\alpha(n)=1, \beta(n)=0$ and $F_{n}$ does not $\tilde{K}$-embed into $X(\beta)$. This also proves that $X(\alpha)$ and $X(\beta)$ are not uniformly homeomorphic whenever $\alpha$ and $\beta$ are not $E_{0}$-equivalent.

\section{REFERENCES}

1. P. G. Casazza, C. L. Garcia and W. B. Johnson, An example of an asymptotically Hilbertian space which fails the approximation property, Proc. Amer. Math. Soc. 129(2001), 3017-3023. MR.1840107 (2002d:46011)

2. P. G. Casazza and N. J. Kalton, Uniqueness of unconditional bases in Banach spaces, Israel J. Math. 103(1998), 141-175. MR.1613564 (99d:46007)

3. S. Dilworth, V. Ferenczi, D. Kutzarova and E. Odell, On strongly asymptotic $\ell_{p}$ spaces and minimality, J. Lond. Math. Soc.(2) 75(2007), 409-419. MR2340235 (2008g:46016)

4. V. Ferenczi and E. M. Galego, Some equivalence relations which are Borel reducible to isomorphism between separable Banach spaces, Israel J. Math. 152(2006), 61-82. MR.2214453 (2007a:03054)

5. V. Ferenczi, A. Louveau and C. Rosendal, The complexity of classifying separable Banach spaces up to isomorphism, J. Lond. Math. Soc.(2) 79(2009), 323-345. MR2496517

6. V. Ferenczi and C. Rosendal, On the number of non-isomorphic subspaces of a Banach space, Studia Math. 168(2005), 203-216. MR2146123 (2006g:46008)

7. V. Ferenczi and C. Rosendal, Ergodic Banach spaces, Adv. Math. 195(2005), 259-282. MR2145797 (2006b:46007)

8. T. Figiel and W. B. Johnson, A uniformly convex Banach space which contains no $\ell_{p}$, Compositio Math. 29(1974), 179-190. MR0355537 (50:8011)

9. S. Gao, S. Jackson and B. Sari, On the complexity of the uniform homeomorphism relation between separable Banach spaces, Trans. Amer. Math. Soc., to appear.

10. W. T. Gowers, A new dichotomy for Banach spaces, Geom. Funct. Anal. 6(1996), 1083-1093. MR.1421876 (97m:46017)

11. W. B. Johnson, A reflexive Banach space which is not sufficienly Euclidean, Studia Math. 55(1976), 201-205. MR0430756 (55:3761)

12. W. B. Johnson, Banach spaces all of whose subspaces have the approximation property, Special Topics of Applied Mathematics (Proceedings Sem. Ges. Math. Datenverarb., Bonn, 1979), North-Holland, Amsterdam, 1980, 15-26. MR585146 (81m:46032)

13. R. Komorowski and N. Tomczak-Jaegermann, Banach spaces without local unconditional structure, Israel J. Math. 89(1995), 205-226. MR.1324462 (96g:46007) 
14. J. Lindenstrauss and L. Tzafriri, Classical Banach spaces. I, Springer-Verlag, Berlin, 1977. MR0500056 (58:17766)

15. G. Pisier, Weak Hilbert spaces, Proc. London Math. Soc. 56(1988), 547-579. MR.931514 (89d:46022)

16. M. Ribe, On uniformly homeomorphic normed spaces, Ark. Mat. 14(1976), 237-244. MR:0440340 (55:13215)

17. C. Rosendal, Incomparable, non-isomorphic and minimal Banach spaces, Fund. Math. 183(2004), 253-274. MR2128711 (2006b:46009)

18. C. Rosendal, Etude descriptive de l'isomorphisme dans la classe des espaces de Banach, These de doctorat de l'Université Paris 6, 2003.

Department of Mathematical Sciences, Lakehead University, Thunder Bay, Ontario, P7B 5E1, CANADA

E-mail address: ranisca@lakeheadu.ca 